TRANSACTIONS OF THE

AMERICAN MATHEMATICAL SOCIETY

Volume 329, Number 1, January 1992

\title{
THE DETERMINATION OF MINIMAL PROJECTIONS AND EXTENSIONS IN $L^{1}$
}

\author{
B. L. CHALMERS AND F. T. METCALF
}

\begin{abstract}
Equations are derived which are shown to be necessary and sufficient for finite rank projections in $L^{1}$ to be minimal. More generally, these equations are also necessary and sufficient to determine operators of minimal norm which extend a fixed linear action on a given finite-dimensional subspace of $L^{1}$ and thus may be viewed as an extension of the Hahn-Banach theorem to higher dimensions in the $L^{1}$ setting. These equations are solved in terms of an $L^{1}$ best approximation problem and the required orthogonality conditions. Moreover, this solution has a simple geometric interpretation. Questions of uniqueness are considered and a number of examples are given to illustrate the usefulness of these equations in determining minimal projections and extensions, including the minimal $L^{1}$ projection onto the quadratics.
\end{abstract}

\section{INTRODUCTION}

In [3] Cheney and Franchetti derived sufficient and necessary (assuming the subspace is "smooth") conditions for finite rank $L^{1}$ projections to be minimal (Theorem 1 below). One of the conditions is an equation (constancy of the Lebesgue function) and the remaining conditions are of an inequality nature. As an application of the sufficiency of these conditions, they obtained the minimal projection from $L^{1}[-1,1]$ onto the lines $[1, t]$. In $\S 2$ we develop a set of easily applicable necessary and sufficient equations, with no assumptions on the subspace. Finally in $\S 2$, we will demonstrate that these equations imply certain linear equations known to be necessary in this context [2]. In $\S 3$ it will be shown that these equations admit a solution in terms of a family of best approximation problems in $L^{1}$ and the orthogonality conditions, a solution which has a simple geometric interpretation. In $\S 4$ we will develop criteria for both uniqueness and nonuniqueness. As applications, in $\S 5$, we will rederive the Cheney-Franchetti example of the minimal projection onto $[1, t]$, and also obtain the minimal projection onto the quadratics $\left[1, t, t^{2}\right]$. We will also give a new proof of minimality in the setting of general compact abelian groups. The equations developed in $\S \S 2$ and 3 (Theorems 2 and 3 ) apply equally well to operators of minimal norm extending any fixed action on the given subspace and thus may be viewed as an extension of the Hahn-Banach theorem to higher dimensions in the $L^{1}$ setting. Examples of this will also be given in $\S 5$.

We note that the existence of the minimal operators, characterized in Theorem 2 in $\S 2$, is known (see, e.g., [8]).

Received by the editors May 15, 1989 and, in revised form, November 8, 1989.

1980 Mathematics Subject Classification (1985 Revision). Primary 41A35; Secondary 41A65.

(C) 1992 American Mathematical Society $0002-9947 / 92 \$ 1.00+\$ .25$ per page 
Theorem 1 (Franchetti and Cheney [3]). Let $(T, \Sigma, \nu)$ be a measure space for which $\left(L^{1}\right)^{*}=L^{\infty}$. Let $\mathscr{V}$ be a finite-dimensional subspace of $L^{1}$, and let $P=\sum u_{i} \otimes v_{i}$ be a projection of $L^{1}$ onto $\mathscr{V}$ with $u_{i} \in L^{\infty}$ and $v_{i} \in \mathscr{V}$. In order that $P$ be minimal, the following two conditions are sufficient; they are also necessary under the additional assumptions that $\mathscr{V}$ is smooth, $\nu(T)<\infty$, and $\nu$ is nonatomic:

(a) the Lebesgue function of $P$ is constant (a.e.), and

(b) there do not exist $\delta_{1}, \ldots, \delta_{n} \in \mathscr{V}^{\perp} \cap L^{\infty}$ such that

$$
\text { ess sup } \sum_{i=1}^{n} \delta_{i}(t) V_{i}(t)<0,
$$

where

$$
V_{i}(t)=\int_{T} v_{i}(s) \operatorname{sgn} k(t, s) d \nu(s) \quad \text { and } \quad k(t, s)=\sum_{j=1}^{n} u_{j}(t) v_{j}(s) .
$$

Recall that, in an $L^{1}$ space, the subspace $\mathscr{V}$ is said to be smooth if and only if each member of $\mathscr{V} \backslash 0$ is almost everywhere different from 0 . For $u \in L^{\infty}$ and $v \in L^{1}$, the tensor notation $u \otimes v$ denotes the linear operator on $L^{1}$ whose value at $f$ is given by $\left(\int f u d \nu\right) v$. Further notation which will be used in the following is

$$
\begin{gathered}
\vec{y}=\left(y_{1}, \ldots, y_{n}\right), \quad \vec{y} \cdot \vec{z}=y_{1} z_{1}+\cdots+y_{n} z_{n}, \\
|\vec{y}|=(\vec{y} \cdot \vec{y})^{1 / 2}, \quad \text { and } \quad \vec{u} \odot \vec{v}=\sum u_{i} \otimes v_{i} .
\end{gathered}
$$

Also, the Lebesgue function $L(t)$ for $P$ is given by

$$
L(t)=\int_{T}|k(t, s)| d \nu(s)=\dot{\vec{u}}(t) \cdot \vec{V}(t), \quad t \in T ;
$$

note that $\|P\|=\operatorname{ess} \sup L(t)$; see [3, Lemma 2]. Throughout this paper the notation $t \in T^{\prime} \subseteq T$ will mean for almost all $t$ out of $T^{\prime}$ relative to the measure $\nu$.

Theorem 1 above may be extended to families of operators which have any fixed action on $\mathscr{V}$, not necessarily just the identity action as in the case of projections. To ascertain the validity of this, straightforward changes can be made in the Franchetti and Cheney paper [3]. The resulting statement is given in the following theorem.

Theorem 1A. Theorem 1 holds where $P=\sum u_{i} \otimes v_{i}$ is no longer necessarily a projection, but an operator where

$$
\int_{T} v_{i}(t) u_{j}(t) d \nu(t)=a_{i j} \quad(i, j=1, \ldots, n),
$$

with the matrix $A=\left(a_{i j}\right)$ fixed, but nonzero.

As an example consider $\mathscr{V}=\left[1, t, t^{2}\right]$ on $T=[0,1]$. Choosing

$$
\vec{v}(t)=\left((1-t)^{2}, 2 t(1-t), t^{2}\right) \text { and } A=\left(\begin{array}{ccc}
1 & \frac{1}{4} & 0 \\
0 & \frac{1}{2} & 0 \\
0 & \frac{1}{4} & 1
\end{array}\right)
$$


corresponds to the action of the Bernstein operator on the quadratics. For other examples where the action on $\mathscr{V}$ is not necessarily the identity, see $\S 5$.

In the following all the statements and results will refer to the operator $P$ (and the associated "action" matrix $A$ ) of Theorem 1A. Note that if $P$ is a projection then $A$ is the identity matrix.

\section{NECESSARY AND SUFFICIENT EQUATIONS}

Throughout the remainder of this paper it will be assumed that $(T, \Sigma, \nu)$ is a complete measure space and that $\nu$ is strictly localizable. This latter condition is satisfied, for example, if $\nu$ is $\sigma$-finite, and is equivalent to the existence of a lifting on $L^{\infty}(\nu)$. The measure $\nu$ being strictly localizable implies that $\nu$ is localizable, which is equivalent to $\left(L^{1}\right)^{*}=L^{\infty}$, and is also equivalent to $\nu$ having the Radon-Nikodym property. For the pertinent definitions and theory see, e.g., [9]. For simplicity we assume also that the underlying field is the real numbers $R$, and note that, with slight modifications, the field can be taken to be the complex numbers instead.

The following theorem provides necessary and sufficient (equality) conditions for the operator $P$ to be minimal.

Theorem 2. Let $(T, \Sigma, \nu)$ be a complete measure space for which $\nu$ is strictly localizable. Let $\mathscr{V}$ be a finite-dimensional subspace of $L^{1}$, and let $P=\vec{u} \odot \vec{v}=$ $\sum_{i=1}^{n} u_{i} \otimes v_{i}$ be an operator mapping $L^{1}$ into $\mathscr{V}$ with $u_{i} \in L^{\infty}(i=1, \ldots, n)$, $\vec{v}=\left(v_{1}, \ldots, v_{n}\right)$ a fixed basis for $\mathscr{V}$, and the matrix $A$ fixed, as in Theorem 1A. In order that $P$ be minimal, the following equality conditions are necessary and sufficient: there exists a nonzero $n \times n$ matrix $M$ such that

(a) the Lebesgue function $L(t)=\|P\|$ on $T^{\prime}=\operatorname{supp}(M \vec{v})$, and

(b) there exists a positive function $\phi$ such that

$$
\phi(t) \vec{V}(t)=M \vec{v}(t), \quad t \in T^{\prime} .
$$

(In fact, $\phi=\vec{u} \cdot M \vec{v} /\|P\|$.)

Proof. We first note that $P$ endowed with its operator norm can be identified with $\vec{u} \odot \vec{v}$ acting as a continuous function on the compact set $K^{\prime}=B\left(\left(L^{\infty}\right)^{*}\right) \times$ $B\left(L^{\infty}\right)$, where $B$ denotes the unit ball, the topology is thie weak* topology in each case, and $\vec{u} \odot \vec{v}(x, y)=\langle x, \vec{u}\rangle \cdot\langle\vec{v}, y\rangle$, where $\left\langle z, z^{*}\right\rangle$ denotes the action of $z^{*} \in Z^{*}$ on $z \in Z$, and $\left\langle z^{*}, z\right\rangle$ is defined to be $\left\langle z, z^{*}\right\rangle$. (Note that $\langle\vec{v}, y\rangle=\int_{T} \vec{v} y d \nu$, and $\langle x, \vec{u}\rangle=\int_{T} x \vec{u} d \nu$ if $x \in L^{1}$.) But, by use of the KreinMilman Theorem (implying $B\left(Z^{*}\right)$ is the closed convex hull of its extreme points), $K^{\prime}$ can be replaced by the compact set $K=\overline{E B}\left(\left(L^{\infty}\right)^{*}\right) \times \overline{E B}\left(L^{\infty}\right)$, where $\overline{E B}$ denotes the weak* closure of the extreme points in $B$.

Let $\mathscr{E}(P)=\{(x, \pm y) \in K ; \vec{u} \odot \vec{v}(x, \pm y)= \pm\|P\|\}$. Let $\Delta=\{\vec{\varepsilon} \odot \vec{v} ; \vec{\varepsilon} \in$ $\left.\left(\mathscr{V}^{\perp}\right)^{n}\right\}$, fix $\vec{u}_{0} \in\left(L^{\infty}\right)^{n}$ such that $\vec{u}_{0} \odot \vec{v}$ is admissible, and let $P=\vec{u}_{0} \odot$ $\vec{v}-\vec{\varepsilon}_{0} \odot \vec{v}$ for a suitable $\vec{\varepsilon}_{0}$. Then applying standard duality theory for best approximation in a continuous function space (see, e.g., [10, Theorem 1.1 (p. 18) and Theorem 1.3 (p. 29)]), we have that $P$ is minimal if and only if there exists a finite, nonzero, signed measure $\mu$ on $\mathscr{E}(P)$ such that $\operatorname{sgn} \mu((x, \pm y))=$ \pm 1 and

$$
0 \equiv \int_{\mathscr{E}(P)} \vec{\varepsilon} \odot \vec{v} d \mu \text { on } \Delta .
$$


Note further that $\mathscr{E}(P)=\mathscr{E}^{+}(P) \cup \mathscr{E}^{-}(P)$, where

$$
\mathscr{E}^{ \pm}(P)=\{(x, \pm y)\}_{(x, \pm y) \in \mathscr{E}(P)} .
$$

Letting $\mu^{+}=\left.\mu\right|_{\mathscr{E}+}$ and $\mu^{-}=-\left.\mu\right|_{\mathscr{E}-}$, and extending $\mu^{ \pm}$to $\mathscr{E} \mp$ by symmetry, we "symmetrize" $\mu$ by setting $\mu_{s}=\left(\mu^{+}+\mu^{-}\right) / 2$ on $\mathscr{E}^{+}$and $\mu_{s}=$ $\left(-\mu^{+}-\mu^{-}\right) / 2$ on $\mathscr{E}-$. Thus, the homogeneity of $\left(\vec{u}_{0}-\vec{\delta}_{0}\right) \odot \vec{v}(x, y)$, as a function of $y$, shows that the signed measure $\mu$ on $\mathscr{E}$ may be replaced by the (nonnegative) measure $\mu_{s}$ on $\mathscr{E}^{+}$, which therefore induces a measure $\mu_{1}$ which is supported on $\mathscr{E}_{1}(P)=\left\{x ;(x,+y) \in \mathscr{E}^{+}(P)\right\}$, since, for $(x,+y) \in \mathscr{E}^{+}(P)$, $y=\operatorname{sgn}(\langle x, \vec{u}\rangle \cdot \vec{v})$ is a function of $x$. Thus, (2) is equivalent to

$$
\begin{aligned}
0 & \equiv \int_{\mathscr{E}_{1}(P)}\langle x, \vec{\varepsilon}\rangle \cdot\langle\vec{v}, y\rangle d \mu_{1}(x) \\
& =\left\langle\int_{\mathscr{E}_{1}(P)}\langle\vec{v}, y\rangle x d \mu_{1}(x), \vec{\varepsilon}\right\rangle \quad \text { for all } \vec{\varepsilon} \in\left(\mathscr{V}^{\perp}\right)^{n}=\left(\mathscr{V}^{n}\right)^{\perp},
\end{aligned}
$$

i.e.,

$$
\int_{\mathscr{E}_{1}(P)}\langle\vec{v}, y\rangle x d \mu_{1}(x)=M \vec{v}
$$

for some $n \times n$ matrix $M$.

Now $E B\left(\left(L^{\infty}\right)^{*}\right)$ is known to consist of the nonzero multiplicative linear functionals on $L^{\infty}$ (see, e.g., [9]) and is easily seen to be a weak* closed subset of independent elements in $\left(L^{\infty}\right)^{*}$. Furthermore, it is easy to check that the mapping $t \rightarrow \delta_{t}^{*}=\delta_{t} \circ \rho$, where $\delta_{t}$ represents point evaluation at $t$ and $\rho$ is a lifting (a linear, multiplicative selection from the equivalence classes) on $L^{\infty}(\nu)$, is a 1-1 mapping from $T_{1}$ into $E B\left(\left(L^{\infty}\right)^{*}\right)$ for some $T_{1} \subset T$, $\nu\left(T_{1}\right)=\nu(T)$. Thus, $\mathscr{E}_{1}(P) \subset\left\{\delta_{t}^{*}\right\}_{t \in T} \cup\left[\mathscr{E}_{1}(P)-\left\{\delta_{t}^{*}\right\}_{t \in T}\right]$ and, viewing $M \vec{v}$ as an element of $\left(\left(L^{\infty}\right)^{*}\right)^{n}$, we can write

$$
\begin{gathered}
\int_{x \in\left\{\delta_{t}^{*}\right\}_{t \in T}}\langle\vec{v}, y\rangle x d \mu_{1}(x)+\int_{x \in \mathscr{E}_{1}(P)-\left\{\delta_{t}^{*}\right\}_{t \in T}}\langle\vec{v}, y\rangle x d \mu_{1}(x) \\
=\int_{t \in T} M \vec{v}(t) \delta_{t}^{*} d \nu(t) .
\end{gathered}
$$

But now, by the independence of the set $\mathscr{E}_{1}(P)$ in $\left(L^{\infty}\right)^{*}$, we conclude that $\mu_{1}$ has no support on $\mathscr{E}_{1}(P)-\left\{\delta_{t}^{*}\right\}_{t \in T}$ and that $\mu_{1}$ therefore induces a measure (call it $\mu_{1}$ again) on $T$ such that

$$
\left\langle\vec{v}, \operatorname{sgn}\left(\left\langle\delta_{t}^{*}, \vec{u}\right\rangle \cdot \vec{v}\right)\right\rangle d \mu_{1}(t)=M \vec{v}(t) d \nu(t) \quad \text { for (almost all) } t \in T .
$$

Hence, $\mu_{1}$ is absolutely continuous with respect to $\nu$ and thus, since $\left\langle\delta_{1}^{*}, \vec{u}\right\rangle=$ $(\rho \vec{u})(t)=\vec{u}(t)$ a.e. $(\nu)$, the proof is concluded. (Recall that $\|P\|=\operatorname{ess} \sup \vec{u}(t) \cdot$ $\vec{V}(t)$, and so $A$ nonzero implies that $[\vec{V}] \neq\{0\}$, and thus $M$ is also nonzero. Also note that $d \mu_{1} / d \nu=\vec{u} \cdot M \vec{v} /\|P\|$.)

Remark. The Cheney-Franchetti theory (Theorems 1 and 1A) can be used to obtain the conclusion of Theorem 2 under the hypotheses of Theorem 1 (namely $\mathscr{V}$ is smooth (in which case $T^{\prime}=T$ in Theorem 2), $\nu(T)<\infty$, and $\nu$ is nonatomic) as indicated in the following sketch. Identify $P$ with $\vec{u} \odot \vec{v}$ acting as a member of $L^{\infty}(D)$, where $D=\left\{\delta_{t}\right\}_{t \in T} \times \overline{E B}\left(L^{\infty}\right)$, since $\|P\|=$ ess $\sup _{d \in D}(\vec{u} \odot \vec{v})(d)$, and best approximate $\vec{u} \odot \vec{v}$ from $\Delta=\left\{\vec{\varepsilon} \odot \vec{v} ; \vec{\varepsilon} \in\left(\mathscr{V}^{\perp}\right)^{n}\right\}$. 
The content of Theorems 1 and $1 \mathrm{~A}$ in this context is that $P$ is minimal if and only if $\vec{u} \odot \vec{v}$ is (a.e. $(\nu))$ constant $(\|P\|)$ on the "diagonal" set $D_{P}=$ $\left\{\left(\delta_{t}, \operatorname{sgn}(\vec{u}(t) \cdot \vec{v})\right)\right\}_{t \in T} \subset D$ and cannot be improved on $D_{P}$ (i.e., have its $L^{\infty}\left(D_{P}\right)$ norm lowered) by adding to $\vec{u} \odot \vec{v}$ an element $\varepsilon \odot \vec{v}$ in $\Delta$. We can now apply duality theory to conclude that $P$ is minimal if and only if there exists a bounded additive set function $\mu \in\left[L^{\infty}(D)\right]^{*}$ such that (i) $\|\mu\|=1$, (ii) $\langle\vec{u} \odot \vec{v}, \mu\rangle=\|P\|$, (iii) $\langle\Delta, \mu\rangle \equiv 0$. But (i) and (ii) show that $\mu$ is supported on $D_{P}$ (if $\vec{u}(t) \cdot\langle\vec{v}, y\rangle=\|P\|$ then $y=\operatorname{sgn}(\vec{u}(t) \cdot \vec{v})$, i.e., we can regard $\mu$ as an element of $\left[L^{\infty}(T, \Sigma, \nu)\right]^{*}$ and thus (iii) is equivalent to $\int_{T} \varepsilon(t) \cdot \vec{V}(t) d \mu(t)=$ 0 , for all $\vec{\varepsilon} \in\left(\mathscr{V}^{\perp}\right)^{n}=\left(\mathscr{V}^{n}\right)^{\perp}$, which implies that $\vec{V}(t) d \mu(t)=M \vec{v}(t) d \nu(t)$ for some nonzero matrix $M ; \mu>0$ follows easily from (i) and (ii).

The following corollary was shown in [3] in the case $\nu$ is nonatomic.

Corollary 1. If $\mathscr{V}$ is smooth, then the Lebesgue function, for the minimal operator $P$ in Theorem 2, is constant on $T$.

Proof. $T^{\prime}=\operatorname{supp}(M \vec{v})=T$ in Theorem 2.

As a further corollary to Theorem 2 , we can derive, in the event that $\vec{u}$ is sufficiently differentiable and $\mathscr{V}$ is smooth, a linear differential equation known to be necessary for $P$ to be minimal. First, we need the following lemma (see [5, p. 291 or 6 , Corollary 1.2]).

Lemma 1. Suppose that $\mathscr{V}$ is an $n$-dimensional smooth subspace, let $\vec{v}=$ $\left(v_{1}, \ldots, v_{n}\right)$ be a basis for $\mathscr{V}$, let $\vec{a}=\left(a_{1}, \ldots, a_{n}\right) \in R^{n}$, and define

$$
f(\vec{a})=\int_{T}|\vec{a} \cdot \vec{v}(s)| d \nu(s) .
$$

Then

$$
\frac{\partial}{\partial a_{i}} f(\vec{a})=\int_{T} v_{i}(s) \operatorname{sgn}[\vec{a} \cdot \vec{v}(s)] d \nu(s) \quad \text { for } \vec{a} \neq 0 .
$$

Corollary 2. Suppose that $\mathscr{V}$ is an $n$-dimensional smooth subspace and $P=$ $\sum_{i=1}^{n} u_{i} \otimes v_{i}$ is minimal in Theorem 2 (with (1) holding), and suppose further that the $\left\{u_{i}\right\}_{i=1}^{n}$ are piecewise differentiable on $T$. Then the $\left\{u_{i}^{\prime}\right\}_{i=1}^{n}$ satisfy the following linear differential equation on $T$

$$
M \vec{v}(t) \cdot \vec{u}^{\prime}(t)=0 .
$$
[2]).

The equation $(*)$ is a known necessary condition for $P$ to be minimal (see

Proof. Since $P$ is minimal, $L$ is constant on $T$ and therefore by Lemma 1 and Theorem 2,

$$
0=L^{\prime}(t)=\sum_{i=1}^{n} \frac{\partial L}{\partial u_{i}} u_{i}^{\prime}(t)=\vec{V}(t) \cdot \vec{u}^{\prime}(t)=M \vec{v}(t) \cdot \vec{u}^{\prime}(t) / \phi(t)
$$

for $t \in T$.

Remark. In the setting of the applications in $\S 5$ below it will be seen that for $P$ minimal the $u_{i}$ 's must be piecewise differentiable whenever the $v_{i}$ 's are piecewise differentiable. 


\section{SOlUtion OF THE NECESSARY AND SUFFICIENT EQUATIONS}

Theorem 3 below provides a formula and a method for calculating $P_{\min }$ via the simultaneous solution of two standard problems. The first of these problems is the solution of a family of best approximation problems in the $L^{1}$ norm (approximating an element of $\mathscr{V}$ by members of an $n$-1-dimensional subspace); while the second problem involves the solution of a system of equations (the orthonormality equations for $P$ ). The proof of this theorem follows immediately from Theorem 2 and the following Lemma 2 . The result of Theorem 3 admits an especially simple geometric interpretation.

Lemma 2. For $t \in \mathscr{C}(P)=\{t ; L(t)=\|P\|\}$ (the critical set of $P=\vec{u} \odot \vec{v}$ ),

$$
\vec{u}(t)=\|P\| \frac{\alpha(t) \vec{V}(t)-\vec{\gamma}^{*}(t)}{\left\|\left[\alpha(t) \vec{V}(t)-\vec{\gamma}^{*}(t)\right] \cdot \vec{v}\right\|_{1}},
$$

where $\alpha(t)$ is any positive scalar and $\vec{\gamma}^{*}(t)$ yields

$$
\min \|[\alpha(t) \vec{V}(t)-\vec{\gamma}(t)] \cdot \vec{v}\|_{1},
$$

subject to $\vec{\gamma}(t) \cdot \vec{V}(t)=0$.

Proof. Fix $t \in \mathscr{C}(P)$, and let $\Gamma=\{\vec{\gamma} ; \vec{\gamma} \cdot \vec{V}(t)=0\}$. There exists a positive scalar $\alpha$ and a vector $\vec{\gamma}^{*} \in \Gamma$ such that $\vec{u}(t)=\alpha \vec{V}(t)-\vec{\gamma}^{*}$. Hence, $0=\vec{\gamma} \cdot \vec{V}(t)=$ $\int_{T}(\vec{\gamma} \cdot \vec{v}) \operatorname{sgn}\left(\alpha \vec{V}(t) \cdot \vec{v}-\vec{\gamma}^{*} \cdot \vec{v}\right) d \nu$, for all $\vec{\gamma} \in \Gamma$; which shows, by the theory of best approximation in $L^{1}$ (see, e.g., [6]), that $\vec{\gamma}^{*} \cdot \vec{v}$ is a best $L^{1}$-approximation to $\alpha \vec{V}(t) \cdot \vec{v}$ from $\{\vec{\gamma} \cdot \vec{v} ; \vec{\gamma} \in \Gamma\}$. Finally, $\|P\|=\|\vec{u}(t) \cdot \vec{v}\|_{1}=\left\|\left[\alpha \vec{V}(t)-\vec{\gamma}^{*}\right] \cdot \vec{v}\right\|_{1}$. That (3) holds for any positive scalar $\alpha(t)$ follows by scaling the numerator and denominator simultaneously, and using the homogeneity of the norm together with the homogeneity of the best approximation operator.

Definition. For a given $n \times n$ matrix $M$ and fixed $t \in T$, let $\vec{\gamma}^{*}(t ; M)$ denote a solution to the $L^{1}$ best approximation problem

$$
\min _{\vec{\gamma}: \vec{\gamma} \cdot M(t)=0}\|[M \vec{v}(t)] \cdot \vec{v}-\vec{\gamma} \cdot \vec{v}\|_{1} .
$$

Remark. For given $M$ and fixed $t \in T$ in problem (4), $[M \vec{v}(t)] \cdot \vec{v}$ is a fixed element of the $n$-dimensional space $\mathscr{V}$, while $\vec{\gamma} \cdot \vec{v}$ belongs to an $n-1$ dimensional subspace described by $\vec{\gamma} \cdot M \vec{v}(t)=0$. Thus, for given $M$ and $t \in T, \vec{\gamma}^{*}(t ; M)$ exists (not uniquely, in general).

Theorem 3. Under the same hypotheses as in Theorem 2, $P=\sum_{i=1}^{n} u_{i} \otimes v_{i}$ is minimal, with norm $\lambda$, if and only if there exists a nonzero $n \times n$ matrix $M$ such that

$$
\vec{u}(t)=\lambda \frac{M \vec{v}(t)-\vec{\gamma}^{*}(t ; M)}{\left\|[M \vec{v}(t)] \cdot \vec{v}-\vec{\gamma}^{*}(t ; M) \cdot \vec{v}\right\|_{1}}, \quad t \in T^{\prime}=\operatorname{supp}(M \vec{v}),
$$

and

$$
\int_{T} u_{i} v_{j} d \nu=a_{i j}, \quad i, j=1, \ldots, n,
$$

where

$$
\lambda=|\operatorname{tr}(M A)| / \int_{T} \frac{M \vec{v}(t) \cdot M \vec{v}(t)}{\left\|[M \vec{v}(t)] \cdot \vec{v}-\vec{\gamma}^{*}(t ; M) \cdot \vec{v}\right\|_{1}} d \nu(t) .
$$


Proof. Apply Theorem 2 to Lemma 2, i.e., replace $\alpha(t) \vec{V}(t)$ by $M \vec{v}(t)$. Finally, expression (c) for $\lambda$ is a direct consequence of (a) and (b). Let the rows of $M$ be denoted by $\vec{m}_{i}(i=1, \ldots, n)$. Then

$$
\begin{aligned}
\vec{u}(t) \cdot M \vec{v}(t) & =\lambda \frac{\left[M \vec{v}(t)-\vec{\gamma}^{*}(t ; M)\right] \cdot M \vec{v}(t)}{\left\|[M \vec{v}(t)] \cdot \vec{v}-\vec{\gamma}^{*}(t ; M) \cdot \vec{v}\right\|_{1}} \\
& =\lambda \frac{M \vec{v}(t) \cdot M \vec{v}(t)}{\left\|[M \vec{v}(t)] \cdot \vec{v}-\vec{\gamma}^{*}(t ; M) \cdot \vec{v}\right\|_{1}}
\end{aligned}
$$

and

$$
\vec{u}(t) \cdot M \vec{v}(t)=\sum_{i=1}^{n} u_{i}(t)\left[\vec{m}_{i} \cdot \vec{v}(t)\right]=\sum_{i=1}^{n} \vec{m}_{i} \cdot\left[u_{i}(t) \vec{v}(t)\right]
$$

Integration of these expressions, together with (b), yields (c).

Remark. The solution $\vec{\gamma}^{*}(t ; M)$ is homogeneous (of degree 1 ) in $M$, as is easily verified by considering (4). Thus, $\vec{u}(t)$ is homogeneous of degree 0 in $M$, so that the matrix $M$ may be normalized without loss. The orthonormality equations (b) are then $n^{2}$ equations for the $n^{2}-1$ remaining elements of $M$ (a linear combination of these equations was used in deriving relation (c) for $\lambda$, hence only $n^{2}-1$ equations are independent).

Geometric interpretation. Regarding the result of Theorem 3, a geometric interpretation of this solution is especially enlightening. The $R^{n}$ norm given by

$$
\|\vec{a}\|=\|\vec{a} \cdot \vec{v}\|_{1} \quad \text { for } \vec{a} \in R^{n}
$$

plays a central role. If $S$ denotes the unit sphere induced by this norm, then, for $t \in T^{\prime}, \vec{u}(t)$ is a point on the $\lambda$-sphere $\lambda S$ such that a tangent hyperplane at that point is perpendicular (in the Euclidean sense) to the direction given by $M \vec{v}(t)$.

The orthogonality conditions, together with the solution for $\vec{u}(t)$, provide a natural successive approximation iteration for the determination of the matrix $M$ and the norm $\lambda$. Suppose, for convenience, that the matrix $M$ is normalized so that $\operatorname{tr}(M A)=1$. Letting

$$
w(t ; M)=\frac{1}{\left\|[M \vec{v}(t)] \cdot \vec{v}-\vec{\gamma}^{*}(t ; M) \cdot \vec{v}\right\|_{1}}
$$

the orthogonality conditions may be written as (where vectors are interpreted as columns and " $\tau$ " denotes transpose)

$$
\int_{T} \vec{u} \vec{v}^{\tau} d \nu=A \quad \text { or } \quad \lambda[M G(M)-\Gamma(M)]=A,
$$

where

$$
G(M)=\int_{T} w(t ; M) \vec{v}(t) \vec{v}^{\tau}(t) d \nu(t)
$$

and

$$
\Gamma(M)=\int_{T} w(t ; M) \vec{\gamma}^{*}(t ; M) \vec{v}^{\tau}(t) d \nu(t) .
$$

Using the fact that $G(\lambda M)=\frac{1}{\lambda} G(M)$ and $\Gamma(\lambda M)=\Gamma(M)$, for $\lambda>0$, and setting $\Lambda=\lambda M$, we are led to two equations to be solved for $\lambda$ and $\Lambda$ :

$$
\lambda[\Lambda G(\Lambda)-\Gamma(\Lambda)]=A \text { and } \operatorname{tr}(\Lambda A)=\lambda .
$$


These equations suggest the iteration

$$
\Lambda^{(n+1)}=\left[\frac{1}{\lambda^{(n)}} A+\Gamma\left(\Lambda^{(n)}\right)\right] G^{-1}\left(\Lambda^{(n)}\right) \quad \text { and } \quad \lambda^{(n+1)}=\operatorname{tr}\left(\Lambda^{(n+1)} A\right),
$$

where $M=\Lambda / \lambda$ if the process converges. The interesting question of when this iteration process converges is not treated here; however, there are several heuristic reasons for expecting this iteration to converge. First, if the unit sphere $S$ coincides with the unit Euclidean sphere in $R^{n}$ (as would be the case if $\|\vec{a} \cdot \vec{v}\|_{1}$ were replaced by $\|\vec{a} \cdot \vec{v}\|_{2}$ and the $\vec{v}$ were taken orthornormal, referred to below as the " $L^{2}$ analogy"), then $\vec{\gamma}^{*}$ is zero as is the matrix $\Gamma$; thus, the term containing $\Gamma(\Lambda)$ might reasonably be expected to be "small" relative to the other terms. Secondly, the Gram matrix $G(\Lambda)$ depends on $\Lambda$ only through the norm of an error term (see the definition of the weight $w$ ), and thus, might reasonably be expected to be "close to constant" relative to the $\Lambda$ term which multiplies it. Also, using the $L^{2}$ analogy to develop good starting values leads to $\Lambda^{(0)}=A / \operatorname{tr}\left(A^{2}\right)$ and $\lambda^{(0)}=1$ (assume that $\Lambda$ is a scalar multiple of $A$ and that $G(A)=I$ to discover these values). Examples illustrating several cases where this process does in fact converge numerically are given in $\S 5$.

\section{UNIQUENESS}

The question of uniqueness for $P_{\min }$ has been investigated by Cheney and Franchetti in [3]. In this section, we will obtain further information concerning uniqueness, although a general statement remains open. "Smoothness" of $\mathscr{V}$ plays a central role in the uniqueness question.

The following easily checked example shows, for $n=1$, exactly when $P_{\min }$ is unique, and also shows, for $n>1$, that "smoothness" is not essential.

Example. Let $\mathscr{V}$ be a space spanned by $n$ functions $v_{i}$ with disjoint supports $T_{i} \quad(i=1, \ldots, n)$, where $T^{\prime}=\bigcup_{i} T_{i}$, and suppose that $A$ is a diagonal matrix. Then a minimal operator is given by $P_{\min }=\sum u_{i} \otimes v_{i}$ where, for $c_{i}=a_{i i} /\left\|v_{i}\right\|_{1}, u_{i}=c_{i} \operatorname{sgn}\left(v_{i}\right) \quad(i=1, \ldots, n)$, and $\left\|P_{\min }\right\|=\max _{1 \leq i \leq n}\left|a_{i i}\right|$. It follows that $\left.u_{i}\right|_{T^{\prime}}$ is unique if and only if $i \in J=\left\{j ;\left|a_{j j}\right|=\left\|P_{\min }\right\|\right\}$. Thus $P_{\min }$ is unique if and only if all $\left|a_{i i}\right|$ are equal $(1 \leq i \leq n)$ and $T^{\prime}=T$. The values $u_{i}(t)$, for $t \in T-\bigcup_{j \in J} T_{j}$ are arbitrary as long as $\int_{T} v_{i} u_{j} d \nu=a_{i j}$ $(1 \leq i, j \leq n)$ and $L(t)=\sum_{i=1}^{n}\left|u_{i}(t)\right|\left\|v_{i}\right\|_{1} \leq\|P\|$.

Note that in the above example, the case where $T$ is a finite interval, the $T_{i}$ are subintervals, and the $v_{i}$ are the characteristic functions of the $T_{i}$, can be described as the case of 0 -splines with fixed knots.

Lemma 3. Suppose $\mathscr{V}$ is smooth. Then $\operatorname{sgn}(\vec{u}(t) \cdot \vec{v})$ is invariant over the set of all $P_{\min }$.

Proof. Suppose that $P_{1}=\sum u_{1 i} \otimes v_{i}$ and $P_{2}=\sum u_{2 i} \otimes v_{i}$ are both minimal. Then using the constancy of the Lebesgue function (Corollary 1), the fact that $P=\frac{1}{2}\left(P_{1}+P_{2}\right)$ is also minimal, and the triangle inequality, we conclude that

$$
\begin{aligned}
\lambda & \equiv \int_{T}\left|\left[\frac{\vec{u}_{1}(t)+\vec{u}_{2}(t)}{2}\right] \cdot \vec{v}(s)\right| d \nu(s) \\
& \leq \int_{T}\left[\frac{\left|\vec{u}_{1}(t) \cdot \vec{v}(s)\right|+\left|\vec{u}_{2}(t) \cdot \vec{v}(s)\right|}{2}\right] d \nu(s) \equiv \lambda .
\end{aligned}
$$


The equality condition for the triangle inequality then gives that, for $t \in T$,

$$
\operatorname{sgn}\left(\vec{u}_{1}(t) \cdot \vec{v}\right)=\operatorname{sgn}\left(\vec{u}_{2}(t) \cdot \vec{v}\right) .
$$

We record two useful consequences of this lemma in the following theorem.

Theorem 4. $P_{\min }$ is unique if

(a) ([3] if $\nu$ is nonatomic) $\mathscr{V}$ is rotund and smooth; or

(b) $\mathscr{V}$ is smooth and $\vec{u}(t) \cdot \vec{v}$ is determined up to a scalar factor by its roots.

Proof. (a) follows from Lemma 3 and the definition of rotundity in the case that $\mathscr{V}$ is $\operatorname{smooth}$, i.e., $\operatorname{sgn}(\vec{a} \cdot \vec{v})=\operatorname{sgn}(\vec{b} \cdot \vec{v})$ implies that $\vec{a} \cdot \vec{v}=c \vec{b} \cdot \vec{v}$ for some constant $c$, for then, in the notation of Lemma $3, \vec{u}_{1}(t)=c(t) \vec{u}_{2}(t)$ and $c(t) \equiv 1$ by constancy and equality of the Lebesgue functions.

(b) follows from Lemma 3 because, for almost all $t, \vec{u}(t) \cdot \vec{v}$ is unique up to a scalar multiple $c(t)$. But then $c(t) \equiv 1$ as in (a).

Theorem 5. Let $\vec{u}(t) \cdot \vec{v}$ be the kernel of $P_{\min }$. For $n \geq 2$, if there exists $T^{\prime} \subseteq T$, with $\nu\left(T^{\prime}\right)>0$, such that

$$
\underset{(t, s) \in T^{\prime} \times T}{\operatorname{essinf}}|\vec{u}(t) \cdot \vec{v}(s)|>0
$$

then $P_{\min }$ is not unique.

Proof. Let $\varepsilon$ be the value of the essential infimum above. There exists a $\vec{\delta} \neq \overrightarrow{0}$ such that $|\vec{\delta}(t) \cdot \vec{v}(s)|<\varepsilon / 2$ for $(t, s) \in T^{\prime} \times T, \vec{\delta}(t)=\overrightarrow{0}$ for $t \in T \backslash T^{\prime}$, $\vec{\delta} \in\left(\mathscr{V}^{\perp}\right)^{n}$ and $\vec{\delta}(t) \cdot \vec{V}(t)=0, t \in T$. For example, pick $T^{\prime \prime} \subseteq T^{\prime}$ such that $\nu\left(T^{\prime \prime}\right)>0$ and $|\vec{v}|<M<\infty$ on $T^{\prime \prime}$, and assume without loss that $V_{1} \not \equiv 0$ on $T^{\prime \prime}$. Then let $\vec{\delta}=w\left(-V_{2}, V_{1}, 0, \ldots, 0\right)$, where $w \in L^{\infty} \cap\left(\left[V_{1} \mathscr{V}, V_{2} \mathscr{V}\right]\right)^{\perp}$, $w$ is supported on $T^{\prime \prime}$ and $0<\|w\|_{\infty}<\varepsilon /\left(2 M \max \left\|V_{i}\right\|_{\infty}\right)$. Then for $P$ with kernel $(\vec{u}+\vec{\delta})(t) \cdot \vec{v}(s)$, we have

$$
\begin{aligned}
\vec{V}(t) & =\int_{T} \vec{v}(s) \operatorname{sgn}[(\vec{u}+\vec{\delta})(t) \cdot \vec{v}(s)] d \nu(s) \\
& =\int_{T} \vec{v}(s) \operatorname{sgn}[\vec{u}(t) \cdot \vec{v}(s)] d \nu(s),
\end{aligned}
$$

i.e., the Lebesgue function $L(t)=(\vec{u}+\vec{\delta})(t) \cdot \vec{V}(t)=\vec{u}(t) \cdot \vec{V}(t)$ coincides with the Lebesgue function for $P_{\min }$.

Conjecture A. The converse of Theorem 5 is true if $\mathscr{V}$ is smooth.

Lemma 4. Suppose that $n \geq 2, \operatorname{rank}(A) \geq 2$, and $\mathscr{V}$ is smooth. Then $\operatorname{dim}[\vec{V}]$ $\geq 2$.

Proof. Suppose that $\operatorname{dim}[\vec{V}]=1$. Then after a suitable change of basis, the matrix $M$ of Theorem 2 may be assumed to have the form

$$
M=\left(\begin{array}{c}
\vec{m}_{1} \\
\overrightarrow{0} \\
\vdots \\
\overrightarrow{0}
\end{array}\right) .
$$

Thus, the best approximation problem given by equation (3) becomes

$$
\min _{\vec{\alpha}}\left\|\left(\left[\vec{m}_{1} \cdot \vec{v}(t)\right] \vec{e}_{1}-\vec{\gamma}\right) \cdot \vec{v}\right\|_{1},
$$


where $\vec{e}_{1}=(1,0, \ldots, 0)$ and $\vec{\gamma}=\left[\vec{m}_{1} \cdot \vec{v}(t)\right] \vec{\alpha}$ with $\vec{\alpha}=\left(0, \alpha_{2}, \ldots, \alpha_{n}\right)$. Since $\vec{m}_{1} \cdot \vec{v}(t)$ factors out of the above expression, it is immediate that the solution $\vec{\alpha}^{*}$ is independent of $t$. Therefore, by Theorem $3, \vec{u}(t)=w(t)\left[\vec{e}_{1}-\right.$ $\left.\vec{\alpha}^{*}\right]$, where $w(t)$ is a scalar-valued function. Hence, $\operatorname{rank}(A)=\operatorname{dim}[\vec{u}]=1$, which is a contradiction.

Lemma 5. Let $\vec{u}(t) \cdot \vec{v}(s)$ be the kernel of $P_{\min }$. Suppose that $n \geq 2, \operatorname{rank}(A) \geq$ 2 , and $\mathscr{V}$ is smooth. Then, for $t \in T, \vec{u}(t) \cdot \vec{v}(s)$ has at least one sign change as a function of $s$.

Proof. Suppose not. Then $\vec{V}$ is constant on some $T^{\prime} \subseteq T$ with $\nu\left(T^{\prime}\right)>0$ and this, together with Lemma 4 and equations (b) of Theorem 2, violates the smoothness of $\mathscr{V}$.

A consequence of Conjecture A and Lemma 5 would be

Conjecture B. $P_{\min }$ is unique if $\mathscr{V}$ is smooth and continuous, and $\operatorname{rank}(A) \geq 2$ if $n \geq 2$.

Lemma 6. If $\mathscr{V}$ is smooth, then the quantities $\bar{V}(t), \phi(t), M$ in equations (b) of Theorem 2 are unique.

Proof. By Lemma 3, $\vec{V}$ is unique, and thus, $\phi(t)$ and $M$ in (1) are unique up to a scalar multiple.

Recall that $S$ denotes the unit sphere in $R^{n}$ induced by $\|\vec{a}\|=\|\vec{a} \cdot \vec{v}\|_{1}$ for $\vec{a} \in R^{n}$.

Theorem 6. $P_{\min }$ is unique if $\mathscr{V}$ is smooth and $S$ has a unique point of tangency on the plane perpendicular (in the Euclidean sense) to the direction at $M \vec{v}(t)$ for all $t \in T$ ( $M$ given in (1)).

Proof. By Lemma 6, equations (b) of Theorem 2 are unique, and therefore the matrix $M$ of Theorems 2 and 3 is uniquely given. Next, observe that the smoothness on $\mathscr{V}$ provides that $T^{\prime}=T$, i.e., $M \vec{v}(t) \neq \overrightarrow{0}$ for $t \in T$. Further, $\vec{\gamma}^{*}(t)$, yielding min $\|M \vec{v}(t)-\vec{\gamma}\|$, lies on the line through the origin perpendicular (in the Euclidean sense) to $M \vec{v}(t)$ and is therefore uniquely determined for all $t$ except for at most a measure zero subset of $T$ by hypothesis. Thus, $\vec{u}$ is unique by (a) of Theorem 3 .

Corollary 3. If $n=2, \operatorname{rank}(A)=2, \mathscr{V}$ is smooth, and $\nu$ is nonatomic, then $P_{\min }$ is unique.

Proof. Lemma 4 shows that $\operatorname{rank}(M)=2$, and this together with the smoothness of $\mathscr{V}$ shows that $M \vec{v}(t)$ is a different element of $R^{2}$ for each $t \in T$. Next, the one-dimensional boundary of any unit ball in $R^{2}$ can have at most countably many (counterclockwise) tangent directions common to more than one point. Hence $S$ is rotund at $M \vec{v}(t), t \in T$ (a.e. $(\nu)$, since $\nu$ is nonatomic). The result then follows from Theorem 6.

Corollary 4. If $n=2, \operatorname{rank}(A)=2, \mathscr{V}$ is smooth, and $\nu$ is nonatomic, then for $t \in T, \vec{u}(t) \cdot \vec{v}(s)=0$ for some $s \in T$.

Proof. The proof is an immediate consequence of Theorem 5 and Corollary 3. 


\section{Applications}

In order to determine minimal projections in the $L^{1}$ setting, two different procedures will be indicated. The direct solution of Theorem 3 requires solving an associated family of geometric problems (or best approximation problems). This process may be bypassed in certain cases by relying on the fundamental Theorem 2. This method is indicated by the following "prescription" for obtaining the minimal projection $P_{\text {min }}$ from $L^{1}[-1,+1]$ onto $\left[v_{1}, \ldots, v_{n}\right]$. Both of these methods will be illustrated in reconstructing the Franchetti-Cheney example of $\mathscr{V}=[1, t]$ on $T=[-1,+1]$ (with Lebesgue measure).

Secondly, we will obtain $P_{\min }$ for an arbitrary two-dimensional unconditional subspace of $L^{1}[-1,+1]$. Next, $P_{\min }$ will be obtained for the quadratics $\left[1, t, t^{2}\right]$ on $T=[-1,+1]$. Finally, an easy application of the theory yields a new proof for $P_{\min }$ in the setting where $T$ is a compact abelian group.

Prescription. $P_{\min }$ from $L^{1}[-1,+1]$ onto $\left[v_{1}, \ldots, v_{n}\right]$.

Let

$$
\widehat{V}_{i}(\vec{x})=\left(\int_{-1}^{x_{1}}-\int_{x_{1}}^{x_{2}}+\cdots+(-1)^{n-1} \int_{x_{n-1}}^{1}\right) v_{i}(s) d s, \quad i=1, \ldots, n .
$$

Keeping in mind equation (b) of Theorem 2, for a given matrix $M$ with nonzero rows $\vec{m}_{i}(i=1, \ldots, n)$, we solve

$$
\frac{\widehat{V}_{i}(\vec{x}(t))}{\vec{m}_{i} \cdot \vec{v}(t)}=\frac{\widehat{V}_{n}(\vec{x}(t))}{\vec{m}_{n} \cdot \vec{v}(t)}, \quad i=1, \ldots, n-1,
$$

for $\vec{x}(t)=\left(x_{1}(t), \ldots, x_{n-1}(t)\right)$, with $-1<x_{1}(t)<\cdots<x_{n-1}(t)<1$ for each $t \in(-1,1)$. Next, let

$$
\sigma(t)=\operatorname{sgn}\left[\frac{\widehat{V}_{n}(\vec{x}(t))}{\vec{m}_{n} \cdot \vec{v}(t)}\right]
$$

and solve the following $n$ linear equations for $u_{1}(t), \ldots, u_{n}(t)$ :

$$
\begin{array}{ccc}
\widehat{V}_{1}(\vec{x}(t)) u_{1}(t)+\cdots+ & \widehat{V}_{n}(\vec{x}(t)) u_{n}(t) & =\lambda \sigma(t) \\
v_{1}\left(x_{1}(t)\right) u_{1}(t)+\cdots+ & v_{n}\left(x_{1}(t)\right) u_{n}(t)= & 0 \\
\vdots & \vdots & \vdots \\
v_{1}\left(x_{n-1}(t)\right) u_{1}(t)+\cdots+v_{n}\left(x_{n-1}(t)\right) u_{n}(t) & = & 0
\end{array}
$$

There are now $n^{2}$ parameters $m_{i j}$ and the norm parameter $\lambda$ to be determined from the $n^{2}$ orthonormalization conditions

$$
\int_{-1}^{+1} u_{i}(t) v_{j}(t) d t=\delta_{i j} \quad(i, j=1, \ldots, n) .
$$

Note that $\sigma(t) \widehat{V}_{i}(\vec{x}(t))$ will be $V_{i}(t)$ of Theorem 2, $i=1, \ldots, n$. However, the homogeneity of the system (6) allows one of the $m_{i j}$ to be normalized to be 1 , leaving exactly $n^{2}$ equations in $n^{2}$ unknowns. Finally, if (8) can be solved for $\lambda>0$ and, for each $t \in(-1,+1), k(t, s)$ changes sign only at $s=x_{i}(t)$ $(i=1, \ldots, n-1)$ then Theorem 2 guarantees that we have $P_{\min }$. 
Remark. System (7) is equivalent to

$$
\left(\begin{array}{ccc}
v_{1}\left(x_{1}\right) & \cdots & v_{n-1}\left(x_{1}\right) \\
\vdots & & \vdots \\
v_{1}\left(x_{n-1}\right) & \cdots & v_{n-1}\left(x_{n-1}\right)
\end{array}\right)\left(\begin{array}{c}
\psi_{1} \\
\vdots \\
\psi_{n-1}
\end{array}\right)=\left(\begin{array}{c}
-v_{n}\left(x_{1}\right) \\
\vdots \\
-v_{n}\left(x_{n-1}\right)
\end{array}\right) \text {, }
$$

where $\psi_{i}=u_{i} / u_{n}(i=1, \ldots, n-1)$, and

$$
u_{n}=\frac{\lambda \sigma}{\widehat{V}_{1}(\vec{x}) \psi_{1}+\cdots+\widehat{V}_{n-1}(\vec{x}) \psi_{n-1}+\widehat{V}_{n}(\vec{x})} .
$$

Note that in the algebraic case $\left[1, t, \ldots, t^{n-1}\right]$, the $(n-1) \times(n-1)$ matrix above is a Vandermonde matrix.

Remark. The above prescription also applies to the more general problem of Theorem 1A where $d t$ is replaced by $d \nu(t)$ and (8) is replaced by

$$
\int_{-1}^{1} u_{i}(t) v_{j}(t) d \nu(t)=a_{i j} \quad(i, j=1, \ldots, n) .
$$

Note 1 . In the case $\mathscr{V}$ is Haar, then $k(t, s)$, as constructed according to the above prescription, indeed changes sign only at $s=x_{i}(t) \quad(i=1, \ldots, n-1)$.

Application 1. $\mathscr{V}=[1, t]_{[-1,1]}$ (Franchetti-Cheney [3]). This example will be developed in two ways in order to illustrate the solution techniques given by Theorems 2 (the prescription above) and 3, respectively.

Consider first the process described by the above prescription. In this case $n=2, \widehat{V}_{1}(x)=2 x$ and $\widehat{V}_{2}(x)=x^{2}-1$. By use of symmetry considerations, equation (6) becomes

$$
\frac{2 x(t)}{1}=\frac{x^{2}(t)-1}{m t}
$$

from which the admissible solution is $x(t)=m t-\operatorname{sgn}(m t) \sqrt{m^{2} t^{2}+1}$. Equations (9) and (10) are then

$$
\begin{aligned}
\psi(t) & =-x(t) \quad\left(=u_{1}(t) / u_{2}(t)\right) \\
u_{2}(t) & =\frac{\lambda \sigma(t)}{2 x(t) \psi(t)+x^{2}(t)-1} \\
\sigma(t) & =\operatorname{sgn} x(t),
\end{aligned}
$$

which give

$$
u_{1}(t)=\frac{\lambda|x(t)|}{1+x^{2}(t)}, \quad u_{2}(t)=-\frac{\lambda \operatorname{sgn} x(t)}{1+x^{2}(t)} .
$$

By use of the symmetry of $u_{1}$ and $u_{2}$, equations (8) become

$$
\int_{0}^{1} u_{1}(t) d t=\int_{0}^{1} t u_{2}(t) d t=\frac{1}{2},
$$

which are identical to equations (8) and (10) of [3], and result in the equation

$$
2 \psi(1)\left[1-\psi^{2}(1)+\psi(1)\right] \log |\psi(1)|+1-\psi^{2}(1)=0
$$

for $\psi(1)$, and hence $m$. It then follows that $\lambda>0$ and, from Note 1 , Theorem 2 guarantees we have $P_{\min }$. 
Furthermore, if we consider the more general problem where equations (11) become $\int_{-1}^{1} u_{i}(t) v_{j}(t) d t=a_{i j} \quad(i, j=1,2)$, then the procedure leads to

$$
\frac{2 x(t)}{1+m_{1} t}=\frac{x^{2}(t)-1}{m_{2}+m_{3} t} \text {. }
$$

It is easily checked that, for any $m_{1}, m_{2}, m_{3}$, there is exactly one admissible root $x(t)$. Equations (1.9) and (1.10) remain unchanged except that now

$$
\sigma(t)=\frac{\operatorname{sgn} x(t)}{\operatorname{sgn}\left(1+m_{1} t\right)}
$$

leading to

$$
\begin{aligned}
& u_{1}(t)=\frac{\lambda|x(t)|}{\left[1+x^{2}(t)\right] \operatorname{sgn}\left(1+m_{1} t\right)}, \\
& u_{2}(t)=-\frac{\lambda \operatorname{sgn} x(t)}{\left[1+x^{2}(t)\right] \operatorname{sgn}\left(1+m_{1} t\right)} .
\end{aligned}
$$

Equations (1.8) become

$$
\begin{array}{ll}
\int_{-1}^{1} u_{1}(t) d t=a_{11}, & \int_{-1}^{1} t u_{1}(t) d t=a_{12}, \\
\int_{-1}^{1} u_{2}(t) d t=a_{21}, & \int_{-1}^{t} t u_{2}(t) d t=a_{22},
\end{array}
$$

which are to be solved for $\lambda, m_{1}, m_{2}, m_{3}$.

As an illustration, take $a_{12}=a_{21}=0$ and $a_{11}>0$. Then symmetry considerations again allow us to take $m_{1}=m_{2}=0$ and we arrive at

$$
2 \psi(1)\left[\frac{a_{11}}{a_{22}}\left(1-\psi^{2}(1)\right)+\psi(1)\right] \ln |\psi(1)|+1-\psi^{2}(1)=0
$$

as the equation generalizing (14) for $\psi(1)$, and hence for $m_{3}$. Equation (12) is easily seen to be solvable if $2 a_{22}>a_{11}$. Also,

$$
\lambda=a_{11} / 2 \int_{0}^{1} \frac{|x(t)|}{1+x^{2}(t)} d t .
$$

Alternatively, using the geometric procedure of Theorem 3, we can solve the Cheney-Franchetti example by determining the unit sphere $\|\vec{a}\|=1$, where the norm is given by

$$
\|\vec{a}\|=\int_{-1}^{1}\left|a_{1}+a_{2} t\right| d t= \begin{cases}\left(a_{1}^{2}+a_{2}^{2}\right) /\left|a_{2}\right|, & \text { for }\left|a_{1}\right| \leq\left|a_{2}\right|, \\ 2\left|a_{1}\right|, & \text { for }\left|a_{1}\right| \geq\left|a_{2}\right| .\end{cases}
$$

The geometry of the sphere shows that attention can be restricted to the curved part of the sphere which is described by $\left|a_{2}\right|=\left(1+\sqrt{1-4 a_{1}^{2}}\right) / 2$, with the tangent vector given by $\vec{\tau}=\left(1,-2 a_{1} / \sqrt{1-4 a_{1}^{2}}\right)$, for $\left|a_{1}\right|<\frac{1}{2}$. By symmetry considerations the matrix $M$ may be taken diagonal with the normalization $\operatorname{tr}(M)=1$ yielding

$$
M \vec{v}(t)=\left(\begin{array}{cc}
1 /(1+m) & 0 \\
0 & m /(1+m)
\end{array}\right)\left(\begin{array}{l}
1 \\
t
\end{array}\right)=\left(\begin{array}{c}
1 /(1+m) \\
m t /(1+m)
\end{array}\right) .
$$


Thus, $\vec{\tau} \cdot M \vec{v}(t)=0$ yielding

$$
a_{1}(t)=\frac{1}{2 \sqrt{1+m^{2} t^{2}}}
$$

and hence,

$$
\vec{u}(t)=\lambda\left(a_{1}(t), a_{2}(t)\right)=\frac{\lambda}{2}\left(\frac{1}{\sqrt{1+m^{2} t^{2}}}, \operatorname{sgn}(m t)+\frac{m t}{\sqrt{1+m^{2} t^{2}}}\right),
$$

which is easily seen to be the same as the answer obtained by the previous method.

Now, we demonstrate the general procedure for determining the constants $m$ and $\lambda$, as indicated at the end of $\S 3$. First we determine $\alpha$ so that $\vec{\gamma}^{*}(t ; M)=$ $M \vec{v}(t)-\alpha \vec{u}(t) / \lambda$ is orthogonal to $M \vec{v}(t)$, i.e., $\alpha=\lambda|M \vec{v}(t)|^{2} / \vec{u}(t) \cdot M \vec{v}(t)$, which yields $\vec{\gamma}^{*}(t ; M)=\left(m t-\operatorname{sgn}(m t) \sqrt{1+m^{2} t^{2}}\right)(-m t, 1)$. The iteration in (5) converges numerically very rapidly to the correct values for a wide range of starting values for $m$ and $\lambda$ (including those suggested in $\S 3$ ). Other numerical schemes may be used to solve for $m$ from a single equation (see Application 2 below).

Application 2. $\mathscr{V}=\left[v_{1}(t), v_{2}(t)\right]$ is any unconditional two-dimensional smooth subspace of $L^{1}[-1,1]$, where $v_{1}>0$ on $[0,1], v_{2}>0$ on $(0,1]$, and $\left(v_{2} / v_{1}\right)(t) \leq\left(v_{2} / v_{2}\right)(1)$ on $(0,1]$. "Unconditional" means that the norm $\|(a, b)\|$ defined by $\int_{-1}^{1}\left|a v_{1}(t)+b v_{2}(t)\right| d t$ satisfies $\|(a, b)\|=\|(|a|,|b|)\|$ and this implies that $v_{1}$ and $v_{2}$ are even and odd functions respectively.

In this case $n=2$ and $\widehat{V}_{i}(x)=\left(\int_{-1}^{x}-\int_{x}^{1}\right) v_{i}(t) d t, i=1,2$. Using symmetry considerations, equation $(6)$ becomes

$$
\frac{\widehat{V}_{1}(x)}{v_{1}(t)}=\frac{\widehat{V}_{2}(x)}{m v_{2}(t)}
$$

where $m>0$ since $v_{1}>0$ and $v_{2}>0$ on $(0,1)$, and hence $\operatorname{sgn} \widehat{V}_{1}=\operatorname{sgn} \widehat{V}_{2}$ on $(0,1)$.

Lemma 2.1. There exists a unique admissible solution $x(t)$ to (2.6) for each nonzero $t \in[-1,1]$.

Proof. Consider

$$
\begin{aligned}
f(x ; t) & =m v_{2}(t) \widehat{V}_{1}(x)-v_{1}(t) \widehat{V}_{2}(x) \\
& =\left(\int_{-1}^{x}-\int_{x}^{1}\right)\left[m v_{2}(t) v_{1}(s)-v_{1}(t) v_{2}(s)\right] d s \\
& =2\left[(\operatorname{sgn} x) m v_{2}(t) \int_{0}^{|x|} v_{1}(s) d s+v_{1}(t) \int_{|x|}^{1} v_{2}(s) d s\right] .
\end{aligned}
$$

Then $f(-1 ; t)=-f(1 ; t)$ for each $t \in[-1,1]$ shows there exists at least one root for each $t \in[-1,1]$. Further, for $0 \leq t \leq 1, f(x ; t)>0$ for $x>0$, while if $-1 \leq x_{1}<x_{2} \leq 0$ we have

$$
f\left(x_{2} ; t\right)-f\left(x_{1} ; t\right)=2 m v_{2}(t) \int_{x_{1}}^{x_{2}} v_{1}(s) d s-2 v_{1}(t) \int_{x_{1}}^{x_{2}} v_{2}(s) d s>0 .
$$


Thus for $0<t \leq 1, f(x ; t)$ has exactly one root at $x(t) \in[0,1]$. (Note that at $t=0, f( \pm 1 ; 0)=0$.)

Equations (9) and (10) are then

$$
\begin{aligned}
\psi(t) & =\frac{-v_{2}}{v_{1}}(x(t)) \quad\left(=u_{1}(t) / u_{2}(t)\right), \\
u_{2}(t) & =\frac{\lambda \sigma(t)}{\widehat{V}_{1}(x(t)) \psi(t)+\widehat{V}_{2}(x(t))}, \\
\sigma(t) & =\operatorname{sgn}\left[\frac{\widehat{V}_{2}(x(t))}{v_{2}(t)}\right]=\operatorname{sgn} x(t)=-\operatorname{sgn} t,
\end{aligned}
$$

which give

$$
\begin{aligned}
& u_{1}(t)=\frac{\lambda v_{2}(x(t)) \operatorname{sgn} x(t)}{v_{2}(x(t)) \widehat{V}_{1}(x(t))-v_{1}(x(t)) \widehat{V}_{2}(x(t))}, \\
& u_{2}(t)=-\frac{\lambda v_{1}(x(t)) \operatorname{sgn} x(t)}{v_{2}(x(t)) \widehat{V}_{1}(x(t))-v_{1}(x(t)) \widehat{V}_{2}(x(t))} .
\end{aligned}
$$

By use of the symmetry of $u_{1}$ and $u_{2}$, equations (8) become

$$
\int_{0}^{1} u_{1}(t) v_{1}(t) d t=\int_{0}^{1} u_{2}(t) v_{2}(t) d t=\frac{1}{2} .
$$

Lemma 2.2. Equations (2.8) can be solved for $\lambda>0$ and $m$ if $\vec{v}$ is continuous at 1 .

Proof. Rewriting (2.8) we have, using form (2.9) and (2.10),

$$
\int_{0}^{1} \frac{-\psi}{\psi \widehat{V}_{1}(x)+\widehat{V}_{2}(x)} v_{1} d t=\frac{1}{2 \lambda} \text { and } \int_{0}^{1} \frac{-1}{\psi \widehat{V}_{1}(x)+\widehat{V}_{2}(x)} v_{2} d t=\frac{1}{2 \lambda} .
$$

Now subtract these two equations to get an equation for $m$ :

$$
G(m)=\int_{0}^{1} \frac{\psi(t) v_{1}(t)-v_{2}(t)}{\psi(t) \widehat{V}_{1}(x(t))+\widehat{V}_{2}(x(t))} d t=0 .
$$

But $\lim _{m \rightarrow 0^{+}} x(t)=-1$ and $\lim _{m \rightarrow+\infty} x(t)=0$. Hence

$$
G\left(0^{+}\right)=-\frac{1}{\widehat{V}_{1}(-1)\left(v_{2} / v_{1}\right)(-1)} \int_{0}^{1} v_{1}(t)\left[\frac{v_{2}}{v_{1}}(t)-\frac{v_{2}}{v_{1}}(1)\right] d t<0
$$

by hypothesis, while $\lim _{m \rightarrow+\infty} x(t)=0$ implies $G(\infty)=-\int_{0}^{1} v_{2}(t) d t / \widehat{V}_{2}(0)$ $>0$.

Application 3. $\mathscr{V}=\left[1, t, t^{2}\right]_{[-1,1]}$. In this case $n=3$ and

$$
\begin{aligned}
\widehat{V}_{1}\left(x_{1}, x_{2}\right)= & 2\left(x_{1}-x_{2}+1\right), \quad \widehat{V}_{2}\left(x_{1}, x_{2}\right)=x_{1}^{2}-x_{2}^{2}, \\
& \widehat{V}_{3}\left(x_{1}, x_{2}\right)=\frac{2}{3}\left(1+x_{1}^{3}-x_{2}^{3}\right) .
\end{aligned}
$$

By use of symmetry considerations, equations (6) become

$$
\frac{2\left[x_{1}(t)-x_{2}(t)+1\right]}{m_{11}+m_{13} t^{2}}=\frac{x_{1}^{2}(t)-x_{2}^{2}(t)}{t}=\frac{\frac{2}{3}\left[x_{1}^{3}(t)-x_{2}^{3}(t)+1\right]}{m_{31}+m_{33} t^{2}} .
$$


By letting

$$
\gamma_{1}=\frac{m_{11}+m_{13} t^{2}}{2 t} \quad \text { and } \quad \gamma_{3}=\frac{m_{31}+m_{33} t^{2}}{\frac{2}{3} t}
$$

equations (3.6) may be rewritten

$$
x_{1}-x_{2}+1=\gamma_{1}\left(x_{1}^{2}-x_{2}^{2}\right), \quad x_{1}^{3}-x_{2}^{3}+1=\gamma_{3}\left(x_{1}^{2}-x_{2}^{2}\right) .
$$

Introducing the variables $y_{1}=x_{1}-x_{2}$ and $y_{2}=x_{1}+x_{2}$ leads to

$$
y_{1}+1=\gamma_{1} y_{1} y_{2} \text { and } y_{1} \frac{3 y_{2}^{2}+y_{1}^{2}}{4}+1=\gamma_{3} y_{1} y_{2},
$$

which reduce to the single quartic equation for $y_{2}$ :

$$
\left(\gamma_{1} y_{2}-1\right)^{2}\left[3 y_{2}^{2}+4\left(\gamma_{1}-\gamma_{3}\right) y_{2}-4\right]+1=0 \text {. }
$$

This equation is then solved yielding admissible $x_{1}$ and $x_{2} \quad\left(-1 \leq x_{1}(t)<\right.$ $\left.x_{2}(t) \leq 1\right)$. The function $\sigma(t)$ (in (7)) is -1 for $|t|<t_{0}$ and +1 for $t_{0}<|t|$, where $\pm t_{0}$ are points where the admissible solutions of the quartic equation switch from one pair of roots to another. The values of $\lambda, m_{11}, m_{13}, m_{31}$, and $m_{33}$ are determined from the five non-trivial orthonormality conditions

$$
\begin{aligned}
& 1=\int_{-1}^{1} u_{1}(t) d t=\int_{-1}^{1} t^{2} u_{3}(t) d t=\int_{-1}^{1} t u_{2}(t) d t \\
& 0=\int_{-1}^{1} t^{2} u_{1}(t) d t=\int_{-1}^{1} u_{3}(t) d t
\end{aligned}
$$

The solution of these equations (for example, by the iteration method of $\S 3$ ) yields $\lambda=1.359484 \ldots, m_{11}=1.071032 \ldots, m_{13}=-0.714964 \ldots, m_{31}=$ $-0.166288 \ldots, m_{33}=1.361491 \ldots, t_{0}=0.457109 \ldots$. Hence, the $x_{i}(t)$ are specified (equation $(3.8)^{\prime}$ ), and (recall Note 1) $P_{\min }=\vec{u} \odot \vec{v}$, where $\vec{u}(t)$ is given by

$$
\left(\begin{array}{ccc}
\widehat{V}_{1}(\vec{x}(t)) & \widehat{V}_{2}(\vec{x}(t)) & \widehat{V}_{3}(\vec{x}(t)) \\
1 & x_{1}(t) & x_{1}^{2}(t) \\
1 & x_{2}(t) & x_{2}^{2}(t)
\end{array}\right)\left(\begin{array}{l}
u_{1}(t) \\
u_{2}(t) \\
u_{3}(t)
\end{array}\right)=\left(\begin{array}{c}
\lambda \sigma(t) \\
0 \\
0
\end{array}\right)
$$

or

$$
\left(\begin{array}{l}
u_{1}(t) \\
u_{2}(t) \\
u_{3}(t)
\end{array}\right)=\frac{3 \lambda \operatorname{sgn}\left(t y_{2}\right)}{y_{1}^{3}-2-3\left(y_{1}^{2}-y_{2}^{2}\right) / 2}\left(\begin{array}{c}
\left(y_{2}^{2}-y_{1}^{2}\right) / 4 \\
-y_{2} \\
1
\end{array}\right)
$$

and $\left\|P_{\min }\right\|=\lambda$. Finally, by Theorem $4(\mathrm{~b}), P_{\min }$ is unique.

Application 4 ([1]; see also [7]). Let $T$ (with "+") be a compact abelian group with Haar measure $\nu, \widehat{T}$ its dual, $N$ a finite part of $\widehat{T}$, and $\mathscr{V}$ the linear hull of the characters $v_{\tau}, \tau \in N$. In this application $\bar{z}$ denotes the complex conjugate of $z$ and $\operatorname{sgn}(z)=\bar{z} /|z|, z \neq 0$. Further, let

$$
\begin{aligned}
& \mathscr{P}=\left\{P=\sum_{\tau \in N} u_{\tau} \otimes v_{\tau}: L^{1}(T) \rightarrow \mathscr{V} ;\right. \\
& \left.\left.P\right|_{\mathscr{V}}=\left.\sum_{\tau \in N} a_{\tau} v_{\tau} \otimes v_{\tau}\right|_{\mathscr{V}} \text { for fixed } a_{\tau}(\tau \in N)\right\} .
\end{aligned}
$$


Then $P_{\min }=\sum_{\tau \in N} a_{\tau} v_{\tau} \otimes v_{\tau}$ is minimal in $\mathscr{P}$ by (1) (where $\phi \equiv 1$ ) since, first, for $\sigma \in N$,

$$
\begin{aligned}
V_{\sigma}(t) & =\int_{T} v_{\sigma}(s) \operatorname{sgn}\left(\sum_{\tau \in N} \bar{a}_{\tau} \bar{v}_{\tau}(t) v_{\tau}(s)\right) d \nu(s) \\
& =\int_{T} v_{\sigma}(s) \operatorname{sgn}\left(\sum_{\tau \in N} \bar{a}_{\tau} v_{\tau}(s-t)\right) d \nu(s) \\
& =v_{\sigma}(t) \int_{T} v_{\sigma}(r) \operatorname{sgn}\left(\sum_{\tau \in N} \bar{a}_{\tau} v_{\tau}(r)\right) d \nu(r) \\
& =m_{\sigma} v_{\sigma}(t),
\end{aligned}
$$

and thus, secondly,

$$
L(t)=\vec{u}(t) \cdot \vec{V}(t)=\sum_{\tau \in N} \bar{a}_{\tau} \bar{v}_{\tau}(t) m_{\tau} v_{\tau}(t)=\sum_{\tau \in N} \bar{a}_{\tau} m_{\tau} \quad\left(\bar{v}_{\tau}(t) v_{\tau}(t)=1\right), t \in T .
$$

Example 1. In the case of $T$ being the circle group, and $N=\{0, \pm 1, \ldots, \pm n\}$ $\subset \widehat{T}=Z$ (the integers), the Fourier projection $([\vec{u}]=\mathscr{V})$ is minimal, and is furthermore unique by Theorem 4(b) (see also [7]).

Example 2. In the case that $T=[0,1]$ with dyadic addition, let $\mathscr{V}$ be the first $n$ Rademacher functions on $T$. Then the Fourier projection $([\vec{u}]=\mathscr{V})$ is minimal, but is not unique for $n$ odd by Theorem 5 , since

$$
\text { ess inf }|\vec{v}(t) \cdot \vec{v}(s)|=\operatorname{essinf}\left|\sum_{i=1}^{n} v_{i}(t+s)\right| \geq 1 \quad\left(\left|v_{i}(r)\right|=1\right) .
$$

\section{REFERENCES}

1. D. L. Berman, On the impossibility of constructing a linear operator furnishing an approximation within the order of the best approximation, Dokl. Akad. Nauk SSSR 120 (1958), 1175-1177. (Russian)

2. B. L. Chalmers, The n-dimensional Hölder equality condition, (submitted).

3. C. Franchetti and E. W. Cheney, Minimal projections in $L^{1}$-space, Duke Math. J. 43 (1976), 501-510.

4. Charles R. Hobby and John R. Rice, A moment problem in $L^{1}$ approximation, Proc. Amer. Math. Soc. 16 (1965), 665-670.

5. Robert C. James, Orthogonality and linear functionals in normed linear spaces, Trans. Amer. Math. Soc. 61 (1947), 265-292.

6. B. R. Kripke and T. J. Rivlin, Approximation in the metric of $L^{1}(X, \mu)$, Trans. Amer. Math. Soc. 119 (1965), 101-122.

7. Pol V. Lambert, On the minimum norm property of the Fourier projection in $L^{1}$ spaces, Bull. Soc. Math. Belg. 21 (1969), 370-391.

8. P. D. Morris and E. W. Cheney, On the existence and characterization of minimal projections, J. Reine Angew. Math. 270 (1974), 61-76.

9. M. M. Rao, Measure theory and integration, Wiley, New York, 1987.

10. I. Singer, Best approximation in normed linear spaces by elements of linear subspaces, Springer-Verlag, Berlin, 1970. 